7 1. School of Biology, University of St Andrews, UK

8 2. Roslin Institute, University of Edinburgh, UK

9

$10 *$ Corresponding Author

11

12

13

14

15

16

17

18

19

20

21

22

23

24 
27 Access to information is a key advantage of grouping. While experienced animals can lead

28 others to solve problems, less is known about whether partially informed individuals can pool

29 experiences to overcome challenges collectively. Here we provide evidence of such 'experience-

30 pooling'. We presented shoals of sticklebacks (Gasterosteus aculeatus) with a two-stage

31 foraging task requiring them to find and access hidden food. Individual fish were either

32 inexperienced, or had knowledge of just one of the stages. Shoals comprising individuals trained

33 in each of the stages pooled their expertise, allowing more fish to access the food, and to do so

34 more rapidly, compared to other shoal compositions. Strong social effects were identified- the

35 presence of experienced individuals increased the likelihood of untrained fish completing each

36 stage. These findings demonstrate that animal groups can integrate individual experience to solve

37 multi-stage problems, and have significant implications our understanding of social foraging,

38 migration, and social systems.

39

40

41

42

43

44

45

46

47 
48 Group-living provides animals with both ready access to valuable social information and the

49 potential, by processing information through social interactions, to achieve solutions to cognitive

50 problems that might lie beyond the reach of lone individuals ${ }^{1-11}$. Information processing by

51 groups can occur via a number of different mechanisms, and distinguishing between these is a

52 key challenge faced by researchers ${ }^{10}$. Such mechanisms include swarm intelligence, facilitation,

53 and pool-of-competence effects. Swarm intelligence refers to improved cognitive performance

54 that stems from distributed, self-organised decision making, with decisions emerging from

55 repeated local interactions between individuals ${ }^{12,13}$. The many-wrongs principle of collective

56 navigation is an example of swarm intelligence. Here, individuals' motivation to follow their

57 varied and imperfect estimates of the correct travel direction interacts with their drive to remain

58 in close proximity to their neighbours, resulting in a group-level compromise on preferred

59 direction that is more accurate than the separate estimates of most individuals ${ }^{14,15}$. A second

60 mechanism, facilitation, occurs when necessary costs such as vigilance for predators are shared

61 among group members, allowing individuals to allocate more effort to other problems, such as

62 searching for resources ${ }^{11}$. Finally, pool-of competence describes effects arising from group size

63 and diversity, with larger groups being statistically more likely to include more experienced,

64 motivated, persistent or bold individuals that are more likely to solve problems and from which

65 others in the group can acquire information ${ }^{10,16}$.

66

67 Our study is concerned with one aspect of the pool-of-competence effect, specifically variation

68 in experience amongst group members. It is likely often the case in nature that within a given

69 group, members will hold different information about the environment, with some individuals

70 possessing relevant experience in solving a particular challenge that other members lack. This 
71 may be especially so in populations with fission-fusion social structure and in those where group

72 fidelity is low, resulting in high turnover of group membership and frequent disbandment and

73 formation of groups. Research has demonstrated that minorities of experienced individuals can

74 lead their uninformed groupmates ${ }^{17-20}$. Here, leadership may emerge as an outcome of the

75 experienced individuals' attraction to the target and the mutual social attraction between these

76 and their naïve groupmates, ${ }^{3,17,21}$ that is without any communication or direct transmission of

77 information about the target from leader to follower occurring. Often, different group members

78 might have partial but complementary information about the component parts of a particular task

79 that can be broken down into a number of 'stages' or elements. They may be familiar with

80 different sections of a navigation route for example, or some may know where to find food while

81 others might know how to access it. Plausibly, groups of animals may be able to overcome such

82 multi-stage problems through social interactions that combines the separately-held information

83 possessed by individuals, allowing them to reach integrative solutions that lie beyond the grasp

84 of single individuals. ${ }^{5}$ Here we set out to test whether groups of partially informed individuals

85 could indeed pool their knowledge about the separate components of a task to solve complex

86 problems in this manner.

88 We presented shoals of sticklebacks (Gasterosteus aculeatus) with a two-stage navigation and

89 foraging task that required them first to locate a hidden patch of food within a mesh feeder box

90 by swimming through a structured environment towards a light cue (stage 1), and then to access

91 the box by swimming through a small hole in order to obtain the food (stage 2). Some subjects

92 were given prior experience of solving the navigation component of the task, and others

93 experience of accessing food from the food-box, but no single fish had prior experience of both 
94 components of the task. We varied the prior experience possessed by individual fish and

95 arranged these into four different combinations, performing ten replicates per combination. One

96 combination consisted solely of individuals with no prior training (neither knowledge of stage 1,

97 nor stage 2). Two combinations consisted of a majority of untrained fish plus a minority of

98 individuals that had been trained to complete either the navigation part of the task, or to access

99 food from the feeder box (either knowledge of stage 1, or stage 2). Finally, one combination

100 consisted of shoals containing equal numbers of untrained, navigation-trained and feeder-trained

101 fish (both knowledge of stage 1, and of stage 2). We predicted that fish in this latter group would

102 access the food patch most rapidly and that more individuals overall would be successful.

103

104 RESULTS

105

106 The composition of the group (treatment) strongly influenced the number and rate of entries to

107 both the goal area and the feeder (Supplementary Fig. 1).

108

109 Numbers of group members entering goal area and feeder

111 More fish entered the green light goal area in groups that contained trained fish (light trained,

112 Wald $Z=7.37, p<0.01$; feeder trained, Wald $Z=3.6, p<0.01$; combined Wald $Z=6.36, p<0.01$ )

113 than in shoals of untrained fish. As predicted, fish in the combined and light-trained groups

114 entered the goal area more often than those in the feeder-trained groups (light trained compared

115 to feeder trained, Wald $\mathrm{Z}=-4.63, \mathrm{p}<0.01$; combined compared to feeder trained Wald $\mathrm{Z}=-4.9$,

$116 \mathrm{p}<0.01$ ). More fish entered the feeder unit in groups with training (light trained, Wald $\mathrm{Z}=3.17$, 
$117 \mathrm{p}<0.01$; feeder trained, Wald $\mathrm{Z}=3.31, \mathrm{p}<0.01$; combined Wald $\mathrm{Z}=7.2, \mathrm{p}<0.01$ ) than in untrained

118 groups. Again as predicted, we observed elevated rates of entry in the combined groups

119 compared to other treatments (light trained, Wald $\mathrm{Z}=-5.07, \mathrm{p}<0.01$; feeder trained, Wald $\mathrm{Z}=-$

$1204.94, \mathrm{p}<0.01$ Figure 1a).

121

122 When we focused only upon the proportion of naive fish from each group that entered the goal

123 area and the feeders we see a similar pattern of results. There was an increased number of entries

124 into the goal area for shoals containing fish with any type of training (light trained, Wald Z=6.23,

$125 \mathrm{p}<0.01$; feeder trained, Wald $\mathrm{Z}=3.07, \mathrm{p}<0.01$; combined, Wald $\mathrm{Z}=4.74, \mathrm{p}<0.01$ ) compared to

126 untrained groups. We also saw an increased rate of entry into the feeder by naive fish in

127 treatments with training (light trained, Wald $\mathrm{Z}=3.01, \mathrm{p}<0.01$; feeder trained, Wald $\mathrm{Z}=1.96$, $128 \mathrm{p}<0.05$; combined, Wald $\mathrm{Z}=4.96, \mathrm{p}<0.01)$.

130 Rate of entry

131 The time of the first fish in each group to enter the green light goal area was lower in the 132 combined and light-trained groups than it was in the feeder-trained and untrained groups (Cox 133 regression: $W$ ald $\mathrm{Z}=4.05, \mathrm{P}<0.001$ and $\mathrm{Wald} \mathrm{Z}=4.39, \mathrm{P}<0.001)$. The entry times of the first fish 134 in the combined group did not differ from that of the light-trained treatment groups (Wald $\mathrm{Z}=$ 135 0.93, $\mathrm{P}=0.35)$. Regarding entry times into the feeder, as predicted, the first fish in the combined 136 group were faster than all of the other treatment groups (untrained, light-trained and feeder137 trained: Wald $\mathrm{Z}=5.42, \mathrm{P}<0.001 ; \mathrm{Wald} \mathrm{Z}=4.13, \mathrm{P}<0.001$; and Wald $\mathrm{Z}=4.16, \mathrm{P}<0.001$ 138 respectively, Figure $1 \mathrm{~b})$. 
139 An identical pattern was seen when we only considered rates of entries by naïve fish from each

140 group into the goal area and the feeders. For goal area entries there was no difference between

141 the light-trained and combined groups in the rate at which untrained fish first entered the goal

142 area (Wald $\mathrm{Z}=0.64, \mathrm{P}=0.52$ ). The first fish from the untrained and feeder-trained groups took

143 longer to enter the goal area compared to the combined groups (Wald $\mathrm{Z}=2.60, \mathrm{P}=0.009$ and

144 Wald $\mathrm{Z}=2.21, \mathrm{P}=0.027$ ). As predicted, the first naïve fish in the combined groups to enter the

145 feeder did so sooner than the first fish from the untrained, light-trained and feeder-trained groups

146 (Wald $\mathrm{Z}=3.73, \mathrm{P}<0.001 ; \mathrm{W}$ ald $\mathrm{Z}=1.96, \mathrm{P}=0.049$ and $\mathrm{W}$ ald $\mathrm{Z}=2.72, \mathrm{P}=0.007$ respectively).

147 Hazard models

149 Following these analyses we also ran two proportional hazard models to understand the factors

150 that contributed to the fish entering each area (Figure 2).

152 We first ran a set of models that analyzed the rate of entry without explicit consideration of the

153 effect of social information. We found that fish who had prior light-training entered the goal area 154 faster than those without it (Wald $\mathrm{Z}=4.62, \mathrm{p}<0.01$ ), and that fish in the presence of conspecifics 155 with light-training entered the goal area faster than those without (Wald $Z=10.37, p<0.01$ ). A 156 similar, but weaker, effect on rate of entering the goal area was observed for feeder-trained fish 157 (Wald $\mathrm{Z}=2.1, \mathrm{p}=0.04$ ), and fish in a group with feeder-trained fish (Wald $\mathrm{Z}=3.15, \mathrm{p}<0.01$ ). We 158 also found that prior feeder-training (Wald $Z=3.09, \mathrm{p}<0.01$ ), but not prior light-training (Wald $159 \mathrm{Z}=1.55, \mathrm{p}=0.12$ ) increased the rate at which fish entered the feeder, and that the presence of 160 feeder trained fish in the group significantly increased the rate at which all fish entered the feeder 
161 (Wald $\mathrm{Z}=2.81, \mathrm{p}<0.01$ ). Interestingly, the presence of light-trained fish significantly reduced the 162 rate at which other fish entered the feeder (Wald $\mathrm{Z}=-2.72, \mathrm{p}<0.01$ ).

164 Extended models that incorporated social information in more complex ways revealed that 165 previous training to approach the green light (Wald $Z=5.67, p<0.01$ ), the number of light-trained 166 fish (Wald $\mathrm{Z}=5.05, \mathrm{p}<0.01$ ), and the number of feeder-trained fish (Wald $\mathrm{Z}=3.19, \mathrm{p}<0.01$ ) all 167 positively increased the rate at which fish entered the goal area. We also found a strong positive 168 effect of having a shoal mate previously enter the goal area in the last 10 seconds (Wald $169 \mathrm{Z}=19.25, \mathrm{p}<0.01)$, but no effect of having had a shoal mate leave in the previous 10 seconds 170 (Wald $\mathrm{Z}=0.48, \mathrm{p}=0.63$ ). Overall, the number of fish in the goal area was associated with a 171 decrease in the rate of entry for other fish to enter the goal area (Wald $Z=-2.79, p<0.01$ ). 172

173 We found a similar pattern of results when analyzing entry of the feeder. Prior feeder training 174 significantly increases the rate that fish enter the feeder (Wald $Z=2.84, p<0.01$ ), and fish are 175 disproportionately more likely to enter the feeder within 10 seconds of a shoal mate doing so 176 (Wald $\mathrm{Z}=12.83, \mathrm{p}<0.01$ ). We did not find a significantly increased rate for fish entering the 177 feeder within 10 seconds of another fish leaving it (Wald $\mathrm{Z}=1.53, \mathrm{p}=0.13$ ). Overall the number of 178 fish currently in the feeder was associated with a decrease in the rate at which other fish entered 179 the feeder (Wald $\mathrm{Z}=-5.39, \mathrm{p}<0.01$ ). Model coefficients for the goal area and the feeder hazard 180 models can be found in Supplementary Tables 2 and 3. 
184 This experiment provides clear evidence of experience-pooling, with groups of partially

185 informed fish integrating their experience to solve a two-stage foraging problem collectively. A

186 greater proportion of group members gained access to the food patch, and did so sooner, in the

187 mixed groups that contained some fish experienced in the navigation part and others in the

188 feeder-access aspect of the task compared to fish in other treatments which contained members

189 experienced in only one or in neither of the two components of the task. Moreover, naïve fish in

190 the mixed groups also benefitted by accessing the food sooner compared to naïve fish in the

191 other groups. Both experience and social information were significant in affecting entries into the

192 goal area, the first stage of the task. We saw that light-trained fish entered the goal area at a

193 greater rate than did untrained fish, as expected, but also that feeder-trained fish did too. This

194 latter effect possibly arose because the fish were able to see the feeder as they came close to the 195 goal area and, having learned an association between the feeder and food, were more motivated

196 to approach it and enter the goal area than were fish untrained in either task. At the group level,

197 fish in the light-, feeder- and combined groups were more likely to enter the goal area compared

198 to those in the untrained treatment group. Fish with prior feeder-training, and those that were

199 grouped with feeder-trained fish entered the feeder at a greater rate, but we also found a negative

200 effect of the presence of light-trained fish upon feeder entries. This may have resulted from the

201 fact that light-trained were given experience of feeding beneath the green lights, but not in the

202 feeder itself- they may therefore have anticipated finding food beneath the lights, causing them

203 to remain in this area, where they attracted other group members, delaying their entry into the

204 feeder. Further analyses that incorporated social transmission in more nuanced ways revealed

205 that a fish entering the feeder or goal area substantially increased the likelihood that other fish

206 did so in the next ten seconds. This is consistent with both past experimental findings analysing 
207 the following behavior of fish ${ }^{22,23}$ and theory predicting that individuals that are both motivated 208 to move towards a particular location and socially attracted to their groupmates may be able to 209 entrain the group and move them towards the target, a principle termed 'leading according to

210 need' ${ }^{17,18,20,21}$ We found no evidence that the rate at which fish entered the goal area increased

211 immediately after a fish left either the feeder or the goal area suggesting that this effect is

212 mediated by following rather than attention to cues or locations. Such following effects were also

213 identified in a study of recruitment of naïve fish to prey patches by experienced shoal-mates by, ${ }^{23}$

214 who termed them 'untransmitted social effects'. Interestingly, in both hazard models we saw that

215 the number of fish in the goal and feeder areas was associated with a decrease in the rate of entry

216 for other fish into those areas. The reason for this effect is unclear. One possibility is that it was

217 due to trained individuals entering the goal or feeder areas sooner, with naïve fish either entering

218 quickly soon after (fish were more likely to enter the goal and feeder areas if a group mate had

219 entered within the last 10 seconds), or else taking much longer to find or access the patch

220 because they had been left behind. Taken together, our analyses when considered alongside the

221 findings other studies ${ }^{17,18,20,21}$, suggest that leadership arising from the balance between goal

222 orientedness and social attraction may be sufficient to generate collective problem solving.

224 Several factors potentially contribute to the ability of groups to process information and solve 225 problems, from facilitation, to pool-of-competence effects to swarm intelligence, with these 226 mechanisms potentially acting concert. ${ }^{10,16}$ For example, among flocks of songbirds, Morand-

227 Ferron \& Quinn ${ }^{16}$ showed that larger groups of naïve birds were more likely to obtain food from

228 novel feeding devices, that the presence in the group of a knowledgeable bird further increased

229 the likelihood of the group accessing food and that larger groups were more likely to contain 
230 such individuals than smaller ones. Moreover, birds had more success when they were in larger

231 groups and when the feeding devices were closer to cover, compared to when they further away,

232 suggesting that facilitation through reduced predation risk might also affect problem solving. In

233 our study we directly manipulated the experience of group members whilst holding group size

234 constant, allowing us to show that information held by the group could be pooled. This approach

235 also allowed us to rule out other mechanisms, although it doesn't discount the possibility that

236 multiple effects might operate together under natural conditions, as was observed by Morand-

237 Ferron \& Quinn. ${ }^{16}$ Our findings suggest that for groups of ecological generalists negotiating

238 variable environments, diversity in experience and a distributed knowledge base across a group

239 may be of critical importance, potentially more-so than the presence of 'omniscient' individuals

240 will full knowledge of the challenges. ${ }^{24}$ Experience pooling might be especially important within

241 populations that exhibit fission-fusion social structures, where at any point current group

242 members might be expected to possess a greater range of experience than those of more stable

243 groups that have travelled and experienced the same conditions together. We anticipate that

244 experience-pooling, underpinned by leader-follower interactions similar to those seen in our

245 study, might be found in groups of animals facing challenges ranging from learning how to

246 exploit novel foods and avoid new predators to navigating between ephemeral resources and

247 tracing long migration routes.

249 METHODS

251 Subjects 
253 Threespine sticklebacks $(\mathrm{N}=360)$ were collected using hand nets from the Kinnessburn steam in

254 St Andrews, UK in July 2012, and housed in the laboratory in groups of 40 in 901 aquaria. Each

255 aquarium contained a layer of sand and artificial plants, and was connected to an external filter.

256 The temperature in the laboratory was held at $8^{\circ} \mathrm{C}$, and the lights were on for 12 hours per

257 day. The fish were fed daily with frozen blood worms, unless otherwise stated below. We used

258 fish measuring $30-35 \mathrm{~mm}$ in length that showed no signs of been in reproductive condition. Fish

259 were not sexed.

260

261 Overview

262

263 The experiment presented 40 groups of 9 fish with a two-stage navigation task. In order to access

264 a food reward, the fish first had to travel to the far end of a large structured arena, where a feeder

265 box containing the food was hidden behind an opaque screen, and to gain access to the food in

266 the feeder box by entering through one of two small holes. The end of the arena with the feeder

267 box and food reward contained two green lights. We tested groups of fish that contained different

268 combinations of individuals trained to approach green lights, trained to enter the feeder box

269 through the target holes, or not trained in either task. Fish that were not trained to the green lights

270 or to the feeder box were nevertheless exposed to these during training, so as to remove any

271 neophobic responses to the stimuli that may otherwise have confounded their behaviour in the

272 experiment proper. The training procedure and two pilot experiments designed to test the

273 efficacy of the training are described in the supplementary material. All procedures were

274 reviewed and approved by the departmental ethics committee. 
278 The arena (Supplementary Figure 2) consisted of a black plastic box measuring 160cm long,

$279100 \mathrm{~cm}$ wide and $40 \mathrm{~cm}$ tall. It contained a $2 \mathrm{~cm}$-deep layer of fine sand, and was filled with water 280 to a depth of $25 \mathrm{~cm}$. The feeder box was placed $10 \mathrm{~cm}$ from one end of the arena, $40 \mathrm{~cm}$ from each 281 long wall. It was suspended $10 \mathrm{~cm}$ above the sand substrate. The feeder box measured $20 \mathrm{~cm}$ long 282 by $10 \mathrm{~cm}$ tall and wide. It consisted of a $2 \mathrm{~mm}$-wide plastic frame around which were stretched a 283 fine nylon mesh. A $2 \mathrm{~cm}$ x $2 \mathrm{~cm}$ square hole was cut in each end of the feeder box, which enabled 284 fish to swim inside and access the food reward (20 dead bloodworms placed in the centre of the 285 feeder box). The use of a mesh feeder had the advantage that olfactory cues emanating from the 286 food would diffuse through the sides, and would not provide an odor gradient leading to the 287 entrance hole. The food was also visible through the mesh walls and floor of the feeder box, 288 leaving the fish highly motivated to solve the task. However, the fish could not find food simply 289 by swimming towards the sight or smell of it, and previous experiments have shown that this 290 arrangement leaves finding the entrance a challenging task. ${ }^{25}$

292 A white plastic screen measuring $40 \mathrm{~cm} \times 40 \mathrm{~cm}$ was placed $10 \mathrm{~cm}$ in front of the feeder box, and $29330 \mathrm{~cm}$ from each of the long walls of the arena. This prevented the group of fish, which began the 294 experiment at the other end of the arena, from being able to see the feeder box. In order to reach 295 it, they had to swim either side of this barrier. Either side of the feeder box we placed a green 296 LED unit (Trimble, Milton Keynes, UK). These consisted of a circle of 24 individual LEDs set 297 within a case with a diameter of $5 \mathrm{~cm}$. A green filter was taped over each LED unit. Each unit 298 was suspended $10 \mathrm{~cm}$ above the surface of the water, $20 \mathrm{~cm}$ either side of the feeder box, and 20 
$299 \mathrm{~cm}$ from each longwall of the arena. The light produced by the LED units was visible to the fish

300 at the far end of the arena at the beginning of the experiment. A high definition webcam

301 (Logitech C920, Logitech International SA, Lausanne, Switzerland) was mounted 80cm above

302 the feeder box. This was used to film the end the arena immediately behind the barrier, which

303 was designated the 'green light goal area'.

305 At the other end of the arena we placed a holding unit constructed from colourless, perforated

306 plastic. This measured $20 \mathrm{~cm} \times 20 \mathrm{~cm}$, and $40 \mathrm{~cm}$ tall. The bottom and top of the holding unit were

307 open. It was placed directly upon the sand substrate, $5 \mathrm{~cm}$ from the back wall, and $40 \mathrm{~cm}$ from

308 each long side wall of the arena. This was used to house the fish at the beginning of the

309 experiment.

311 In the middle section of the arena we placed four artificial plants. These measured approximately

$31210 \mathrm{~cm}$ tall and $10 \mathrm{~cm}$ in diameter. One pair of plants were placed $20 \mathrm{~cm}$ apart, $30 \mathrm{~cm}$ from each

313 longwall of the arena, and $40 \mathrm{~cm}$ from the end of the arena where the fish holding unit was

314 placed. The second pair of plants were placed $20 \mathrm{~cm}$ from these, and $50 \mathrm{~cm}$ from the white plastic

315 barrier. The plants provided cover for the fish once they were released into the main arena at the

316 beginning of the trial, and facilitated movement throughout the centre of the arena.

318 Experimental groups

320 Fish were allocated using a random number algorithm to replicate groups in four treatments that

321 differed in the experience (i.e. prior training) possessed by constituent members. Each group 
322 contained nine fish, and we ran 10 replicates in each of the four treatments. The first treatment

323 consisted of groups of nine naïve (i.e. non-trained) fish. In the second, each group contained

324 three fish that had been trained to approach the green light and six naïve fish. The third treatment

325 comprised shoals that contained three fish that had been trained to enter the feeder box and six

326 naïve fish, and the fourth contained shoals with three fish from each training regime plus three

327 naïve fish. Hereafter, these treatment groups respectively are referred to as untrained, light-

328 trained, feeder-trained and combined. For clarity, individual fish that had not been trained are

329 referred to as naïve, while the treatment consisting entirely of naïve fish is referred to as

330 untrained. Because familiarity has been shown to affect social foraging interactions in this

331 species, ${ }^{26}$ within each group each fish was drawn from a separate holding tank, ensuring that all

332 were equally unfamiliar to one another. Within each group, every fish was fitted with a non-

333 invasive, colour-coded circular tag on its first dorsal spine. ${ }^{27}$ These were fitted on the last day of

334 training, and the day before the experiments were performed. This allowed us to recognise each

335 individual fish in the videos. Sample sizes were informed by an earlier social foraging

336 experiment conducted in our laboratory. ${ }^{26}$

338 Experimental procedure

340 For each trial, the experimental arena was established as above, and food items (20 dead

341 bloodworms) added to the feeder box. The experimental group was added to the holding unit and

342 allowed to acclimate for 15 minutes, before the holding unit was raised $15 \mathrm{~cm}$ using a pulley,

343 releasing the fish and beginning the trial. The trial ran for a further 45 minutes. From the

344 webcam footage we recorded the identity of each fish as it entered the green light goal area. For 
345 every second of the trial we recorded whether each fish was inside or outside the goal area and

346 inside or outside of the feeder box. We performed five such trials each day (see Supplementary

347 Table 1 for schedule). Following each trial we replaced the water and sand substrate and feeder

348 box prey in the arena. The experimenter was not blind with respect to treatment group.

350 Data availability

352 The datasets analysed during the current study are available from the corresponding author on 353 request.

355 Statistical analysis

357 We analyzed the total proportion of fish and the proportion of untrained fish that entered the 358 green light goal area and the feeder box during the trial using a binomial model. We examined 359 the time at which the first fish entered each area for different groups using Cox regressions, 360 focusing upon the entry times of the first fish (irrespective of training) and the first naive fish 361 from each group. We then used Cox proportional hazard models to model all entries in the group 362 and gain a finer temporal resolution of the factors that predict whether and when fish enter either 363 the goal area or the feeder, and the frequency at which they enter the areas. We examined the rate 364 at which fish entered the feeder and goal area predicted by their previous training, previous time 365 spent in the goal area during the trial, the number of light trained fish, the number of feeder 366 trained fish, and three social cues: the number of fish that had entered the goal/feeder area in the 367 last 10s, the number of fish that had exited the goal/feeder area in the last 10 s, and the total 
368 number of fish in the goal/feeder area. Our data met the assumption of proportional hazards

369 expected by these tests. All proportional hazard models were run in $\mathrm{R}^{28}$ using the "survival"

370 package. $^{29}$

371

372

373 
376 1. Krause, J. \& Ruxton, G. D. Living in Groups. Oxford University Press. (2002).

378 2. Danchin, É. et al. Public information: from nosy neighbors to cultural evolution. Science 305, $379 \quad 487-491(2004)$. 382 Nature 433, 513-516 (2005).

3. Couzin, I. D. et al. Effective leadership and decision-making in animal groups on the move.

391 7. Laland, K. N., Atton, N. \& Webster, M. M. From fish to fashion: experimental and theoretical 392 insights into the evolution of culture. Phil. Trans. R. Soc. Lond. B: Biol. Sci. 366, 958-968 393 (2011).

395 8. Ward, A. J. et al. Fast and accurate decisions through collective vigilance in fish shoals. Proc. 396 Nat. Acad. Sci. 108, 2312-2315 (2011). 
398 9. Berdahl, A. et al. Emergent sensing of complex environments by mobile animal groups.

399 Science 339, 574-576 (2013).

400

401 10. Ioannou, C.C. Swarm intelligence in fish? The difficulty in demonstrating distributed and

402 self-organised collective intelligence in (some) animal groups. Behav. Process. doi:

403 org/10.1016/j.beproc.2016.10.005 (2016).

404

405 11. Ward, A. J. W. \& Webster, M. M. Sociality: The Behaviour of Group Living Animals.

406 Springer International Publishing, Switzerland. (2016).

407

408 12. Bonabeau, E., Dorigo, M. \& Theraulaz, G. Swarm Intelligence: From Natural to Artificial 409 Systems (No. 1). Oxford University Press. (1999).

410

411 13. Garnier, S., Gautrais, J. \& Theraulaz, G. The biological principles of swarm intelligence.

412 Swarm Intel. 1, 3-31 (2007).

413

414 14. Codling, E. A., Pitchford, J. W. \& Simpson, S.D. Group navigation and the "many-wrongs 415 principle' in models of animal movement. Ecology 88, 1864-1870 (2007).

416

417 15. Codling, E. A. \& Bode, N. W. Balancing direct and indirect sources of navigational

418 information in a leaderless model of collective animal movement. J. Theor. Biol. 394, 32-42

419 (2016). 
421 16. Morand-Ferron, J. \& Quinn, J. L. Larger groups of passerines are more efficient problem 422 solvers in the wild. Proc. Nat. Acad. Sci. 108, 15898-15903. (2011).

423

424 17. Dyer, J. R. et al. Leadership, consensus decision making and collective behaviour in humans.

425 Phil. Trans. R. Soc. Lond. B: Biol. Sci. 364, 781-789. (2009).

426

427 18. Ioannou, C. C., Singh, M. \& Couzin, I. D. Potential leaders trade off goal-oriented and 428 socially oriented behavior in mobile animal groups. Am. Nat. 186, 284-293. (2015).

430 19. Jolles, J. W. et al. The role of social attraction and its link with boldness in the collective 431 movements of three-spined sticklebacks. Anim. Behav. 99, 147-153. (2015).

433 20. Webster, M. M. Experience and motivation shape leader-follower interactions in fish shoals. 434 Behav. Ecol. doi: 10.1093/beheco/arw133 (2016).

436 21. Conradt, L. et al. 'Leading according to need' in self-organizing groups. Am. Nat. 173, 304437 312. (2009).

439 22. Day, R. L. et al. Interactions between shoal size and conformity in guppy social foraging. 440 Anim. Behav. 62, 917-925. (2001). 
442 23. Atton, N. et al. Information flow through threespine stickleback networks without social

443 transmission. Proc. R. Soc. Lond. B: Biol. Sci. rspb20121462. (2012).

444

445 24. Krause, S. et al. Swarm intelligence in humans: diversity can trump ability. Anim. Behav. 81, 446 941-948. (2011).

448 25. Reader, S. M. \& Laland, K. N. Diffusion of foraging innovations in the guppy. Anim. Behav.

449 60, 175-180. (2000).

450

451 26. Atton, N. et al. Familiarity affects social network structure and discovery of prey patch

452 locations in foraging stickleback shoals. Proc. R. Soc. Lond. B: Biol. Sci. 281, 20140579. (2014).

453

454 27. Webster, M. M., \& Laland, K. N. Evaluation of a non-invasive tagging system for laboratory 455 studies using three-spined sticklebacks (Gasterosteus aculeatus). J. Fish Biol. 75, 1868-1873.

456 (2009).

457

458 28. R Core Team. R: A Language and Environment for Statistical Computing. Vienna: $\mathrm{R}$

459 Foundation for Statistical Computing. (2013).

460

461 29. Therneau, T. M. \& Lumley, T. Package 'survival'. http://www.r-project.org. (2015).

462

463

464 
467 This work was funded by an ERC Advanced grant to KNL (EVOCULTURE, Ref: 232823). We 468 thank Katherine Meacham for assistance in preparing the manuscript.

470 AUTHOR CONTRIBUTIONS

471

472 MMW designed and performed the experiments, MMW, AW \& KNL analysed the data and co473 authored the paper.

474

475 COMPETING FINANCIAL INTERESTS

476

477 We declare no competing financial interests.

478

479 MATERIALS \& CORRESPONDENCE

480

481 Mike Webster, School of Biology, Harold Mitchell Building, University of St Andrews, Fife

482 KY16 9TS. Email: $\underline{\text { mmw1@ @st-andrews.ac.uk }}$

483

484

485

486

487 
489 Figure 1. (a) The number of fish in each group to enter the green light goal area and the feeder 490 (mean $+/-95 \%$ CI). (b) Survival plots showing the time for the first fish in each group to enter 491 the goal area and feeder. 9U: 9 untrained fish; 3L,6U: 3 light-trained and 6 untrained fish;

492 3F,6U: 3 feeder-trained and 6 untrained fish; 3L,3F,3U, 3 feeder-trained, 3 light-trained and 3 493 untrained fish.

Figure 2. (a) The proportion of fish for each level of training to enter the green light goal area

496 broken down by treatment group. (b) The proportion of fish for each level of training to enter the 497 feeder area broken down by treatment group (c) The proportion of fish for each level of training 498 who entered the goal area and then entered the feeder area broken down by treatment group. In 499 each case, mean +/- 95\% CI is shown. 9U: 9 untrained fish; 3L,6U: 3 light-trained and 6 500 untrained fish; 3F,6U: 3 feeder-trained and 6 untrained fish; 3L,3F,3U, 3 feeder-trained, 3 light501 trained and 3 untrained fish. 
(a) Proportion of fish entering zone
(i) Goal zone
(ii) Feeder

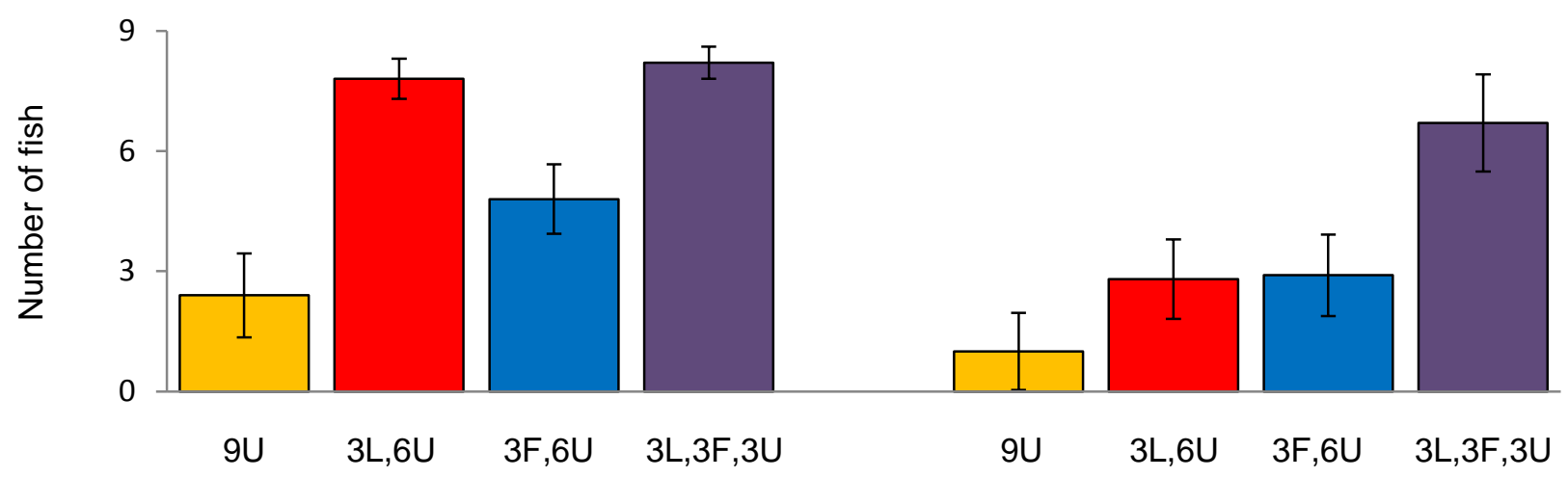

(b) Time for first fish to enter zone

(I) Goal zone

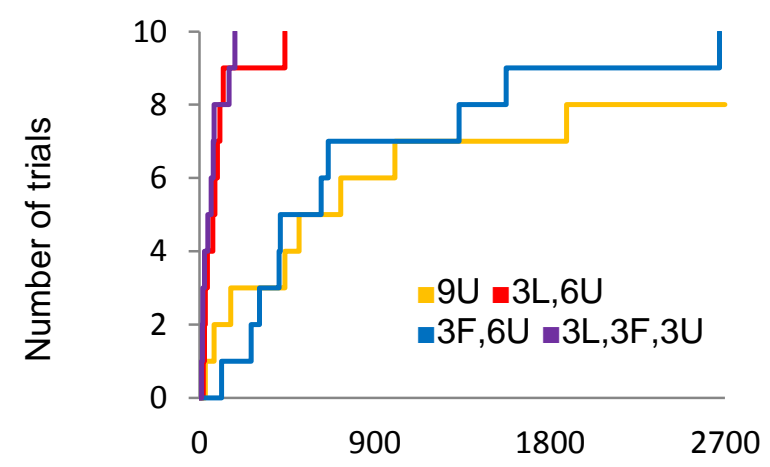

(ii) Feeder

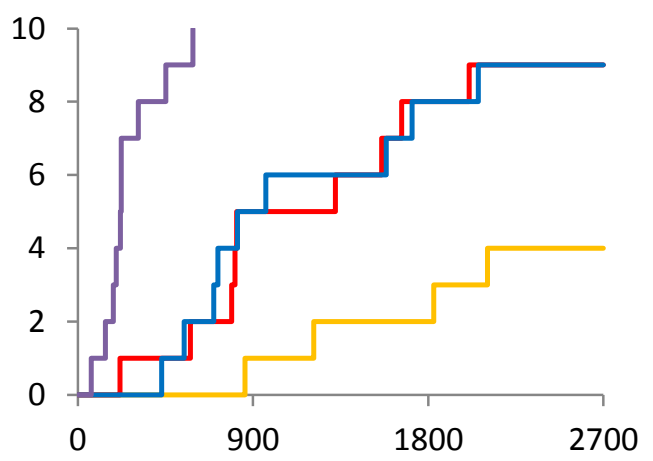


(a) Goal area

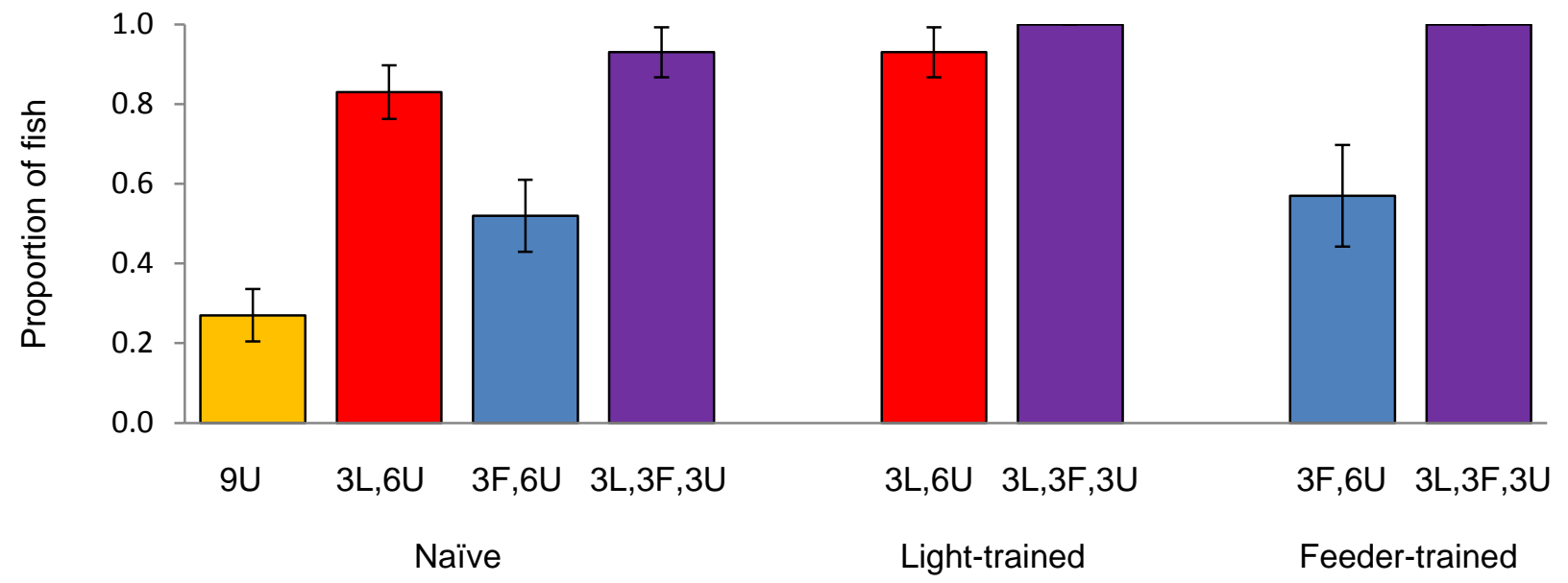

(b) Feeder area

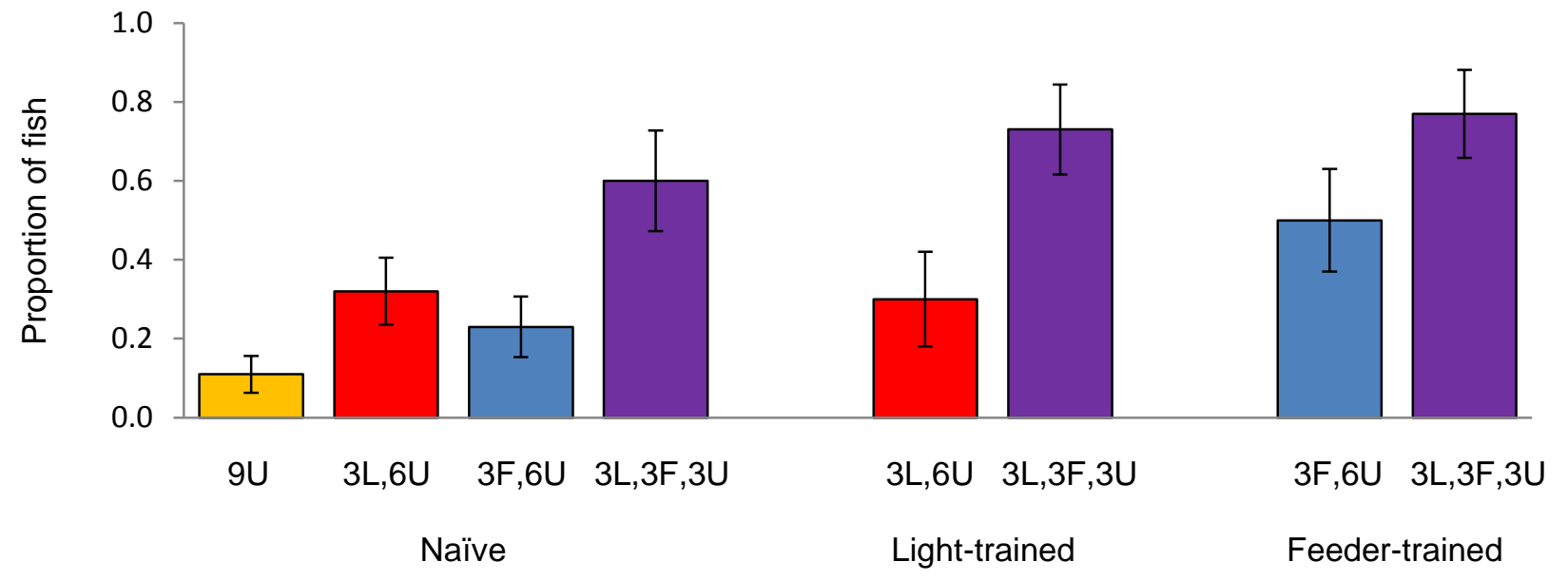

(c) Feeder area given goal area

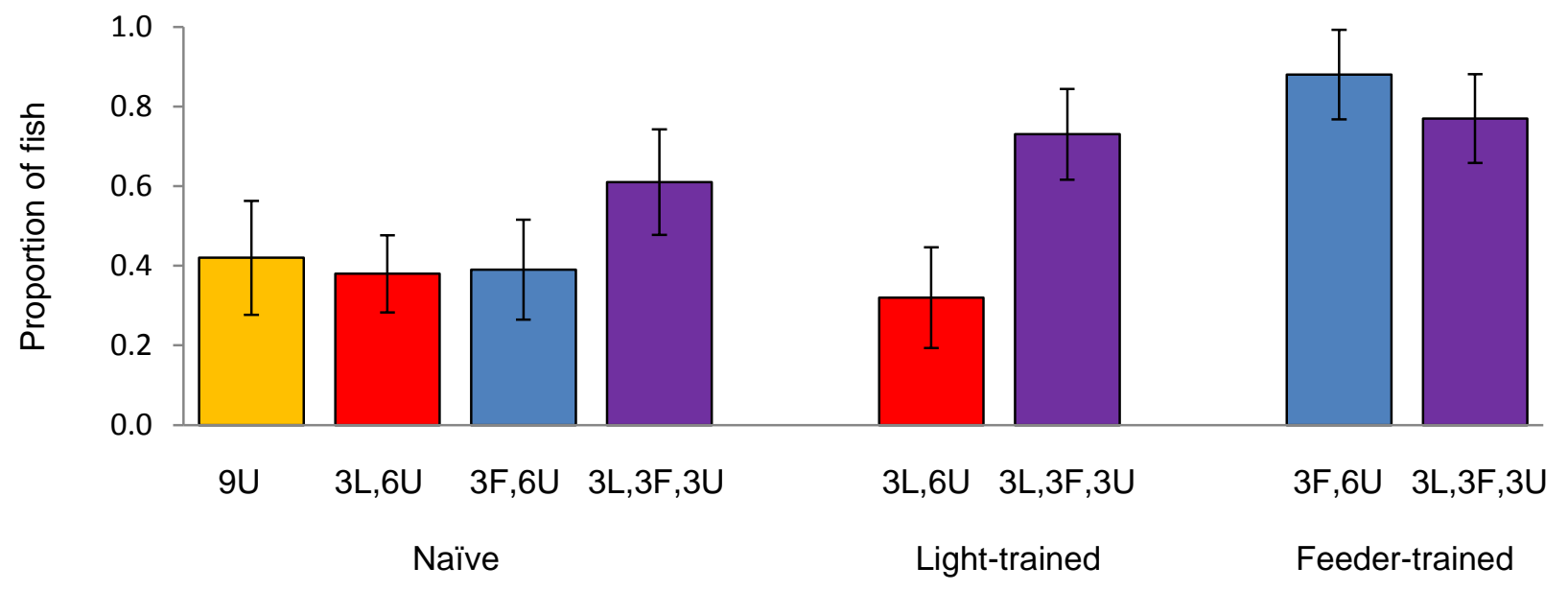


533 Supplementary information for:

534

535

536

537

538

539

540

541

542

543

544

545

546

547

548

549

550

551

552

553

554

555

556

557

558

559

560

561

562

563

564

565

566

567

568

569

\section{Fish pool their experience to solve problems collectively}

Mike M. Webster, Andrew Whalen \& Kevin N. Laland

Contents

1. Supplementary Methods

2. Supplementary Figures 1-4

3. Supplementary Tables 1-3 
572 Training fish to approach the green lights and to access the feeder box

573

574 Prior to beginning the experiments it was necessary to first train the fish. Some fish were trained

575 to approach the green light, some were trained how to enter the feeder box and other fish were

576 not trained to either task. The fish were trained in six batches (Supplementary Table 1). The fish

577 from batches one and two were used in pilot experiments designed to assess the efficacy of the

578 training, with the fish from the third to sixth batches being used in the experiment proper.

580 In batch one and two we set up nine aquaria. Batches three to six contained 18 aquaria. These

581 were sub-divided into two sets of nine aquaria each (referred to as a and b in Supplementary

582 Table 1), with the training and testing regimes in the first set running one day ahead of those in

583 the second. This allowed us to split the experimental trials over two days. Each aquarium

584 contained 10 fish. Only five fish from each aquarium were randomly selected for use in the

585 experiments. We trained additional fish because we anticipated that we would lose some to

586 mortality over the course of training. In fact, none of the trained fish died, but we did not have

587 time to test them all. Untested fish were retained in the laboratory for use in a separate

588 experiment.

590 Each aquarium had a volume of 451 and contained a $2 \mathrm{~cm}$ deep layer of fine sand, and was

591 equipped with an external filter. The aquaria were visually and chemically isolated from one

592 another. The training procedure lasted for four weeks. During the first week the fish were 
593 allowed to acclimate. They were fed daily with frozen bloodworms and were not exposed to the 594 green lights or to the feeder box during this time. At the beginning of the second week training 595 began.

597 In batches one and two, three aquaria were randomly selected and assigned to green light

598 training, three to feeder box training, and three were not trained in either task. In batches three to 599 six, which contain 18 aquaria overall, each subset of nine aquaria was randomly assigned to one 600 of the four experimental treatments (described in main text), such that all nine aquaria received 601 no training, three were trained to the green light while six received no training, three were trained 602 to the feeder while six received no training or three were trained to the feeder, three to the light 603 and three received no training. See Supplementary Table 1 for an overview of the training order 604 and schedule. Fish that were not trained to the green lights or to the feeder box were nevertheless 605 exposed to these, so as to remove any neophobic responses to the stimuli that may otherwise 606 have confounded their behaviour in the experiment proper.

608 A pair of green lights identical to those used in the experimental arena described above were 609 fitted to the end of each aquarium. These were switched on for 15 minutes twice per day at 10am 610 and $4 \mathrm{pm}$. In the aquaria where fish were trained to approach the green lights, food was provided 611 directly beneath the lights at the same time they were switched on. The food was always

612 consumed within the 15 minute period during which the lights were on. In the aquaria where the

613 fish were not trained to associate the lights with food, the lights were kept off during the two

614 daily feeding periods, and were only switched on for 15 minutes one hour after the fish had been

615 fed, and after they had consumed all of the food. Training was repeated daily for three weeks. 
617 In each aquarium we also placed a feeder box, as described above. This was suspended $10 \mathrm{~cm}$

618 above the bottom of the tank. In the aquaria where fish were trained to access the feeder box,

619 training was structured as follows. During the first week they were presented with a feeder box

620 in which both ends had been removed. Food was placed within the feeder box twice per day at

621 10am and 4pm. The fish were easily able to access the food by swimming into the feeder box

622 through the open ends. During the second week, the feeder box was replaced with one with $5 \mathrm{~cm}$

623 square holes in either end, with food placed inside as before. During the third week the feeder

624 box was replaced with one with $2 \mathrm{~cm}$ square holes, identical to the one used in the experiment

625 itself. In both the second and third weeks fish were seen to readily enter the feeder box and eat

626 the food. In the aquaria where the fish were not trained to this task, we used feeder boxes with

627 completely closed ends. For these groups food was provided directly on the sand substrate

628 beneath the feeder box. The fish in these groups had no experience of entering the feeder boxes

629 and no experience of detecting food within them.

\section{Training: pilot experiments}

633 Fish were tested individually in an experimental arena measuring $45 \mathrm{~cm}$ long by $30 \mathrm{~cm}$ tall and 634 wide. The arena was screened in black plastic and contained a $2 \mathrm{~cm}$ deep layer of fine sand, and 635 was filled with water to a depth of $25 \mathrm{~cm}$. At one end of the arena we placed a holding unit 636 measuring $5 \times 5 \mathrm{~cm}$ wide, and $35 \mathrm{~cm}$ tall. This was constructed from colourless perforated plastic.

637 It was open at the top and bottom, and was placed directly upon the sand substrate. A high- 
638 definition WebCam was fixed directly above the experimental arena. This was used to record the

639 trials. Five such arenas were established, allowing five trials to be run simultaneously.

641 We performed two pilot experiments, one in which fish were given the opportunity to approach

642 two green lights located at one end of the tank, and one in which they were presented with a

643 feeder box containing 20 dead blood worms. The lights and the feeder box were as described for

644 the experiment proper, above. We tested the fish from batch one in the green light pilot

645 experiment and those from batch two in the feeder box pilot experiment. Of the 10 fish in each

646 training aquarium, we randomly selected five to be tested. For each pilot experiment we tested

647 three treatment groups (the fish trained to the green light, fish trained to the feeder box and fish

648 that were trained to neither), with 15 replicates in each treatment group. They were tested on the

649 day immediately following the end of the training period.

651 In the green light pilot experiment, two green lights were suspended $10 \mathrm{~cm}$ above the surface of

652 the water at the end of a tank directly opposite the holding unit (Supplementary Figure 3a). No

653 prey were present in the tank in this experiment. The holding unit was used to contain the test

654 subject at the start of the trial. A fish was randomly selected, and carefully transferred from its

655 training aquarium to the holding unit in the experimental arena. It was allowed to acclimate for

65610 minutes. During this period the green lights were switched off. The lights were then switched

657 on and the fish was allowed to settle for another 10 minutes. Following this, the holding unit was

658 carefully raised and removed, releasing the fish and beginning the trial. The trial lasted for a

659 further 10 minutes. From the videos of the trials, we recorded the latency of each fish to enter a

$66010 \mathrm{~cm}$ wide goal zone beneath the lights. 
662 In the feeder box pilot experiment, we suspended a feeder box $10 \mathrm{~cm}$ above the substrate and

$66310 \mathrm{~cm}$ from the back wall of the arena (Supplementary Figure 3b). The feeder box was accessible

664 via two $2 \times 2 \mathrm{~cm}$ holes, identical to the one described above, and as used in the experiment proper.

665 The feeder box contained 20 dead bloodworms. These were added to the feeder box immediately

666 before the fish was added to the holding unit. The fish was allowed to acclimate for 20 minutes

667 before the holding unit was carefully raised and removed, beginning the trial. The trial lasted for

66810 minutes. We recorded the latency of the fish to enter the feeder box.

$670 \quad$ Statistical analyses

672 In the green light and feeder box pilot experiments respectively we compared the latency of the

673 fish to enter goal zone beneath the lights or to enter the feeder box. We used Cox regressions to

674 compare the performance of the fish trained to the green light, to the feeder box and fish that

675 were trained to neither, using the untrained fish as a reference category for an indicator contrast.

677 Pilot experiment results

679 In the green light pilot experiment fish that had been trained to associate the green light with

680 food approached it sooner than did the untrained fish (Wald $X^{2}=21.24, \mathrm{df}=1, \mathrm{P}<0.001$ ), while the

681 feeder-trained fish were no faster than the untrained fish $\left(X^{2}=0.91, \mathrm{df}=1, \mathrm{P}=0.34\right.$ Supplementary

682 Figure 4a). In the feeder box pilot experiment it was the feeder box-trained that entered it sooner

683 than the untrained fish (Wald $X^{2}=18.06, \mathrm{df}=2, \mathrm{P}<0.001$ ), while the light-trained fish and the 
684 untrained fish did not differ $\left(X^{2}=0.81, \mathrm{df}=1, \mathrm{P}=0.37\right.$ Supplementary Figure $\left.4 \mathrm{~b}\right)$. In this

685 experiment, while all of the feeder box-trained fish entered the feeder during the trial, only four

686 of the green light-trained and two of the untrained fish (out of fifteen) entered feeder box. Based

687 on these findings we determined that the two training protocols had been effective.

688 


\section{(a) 9 untrained}

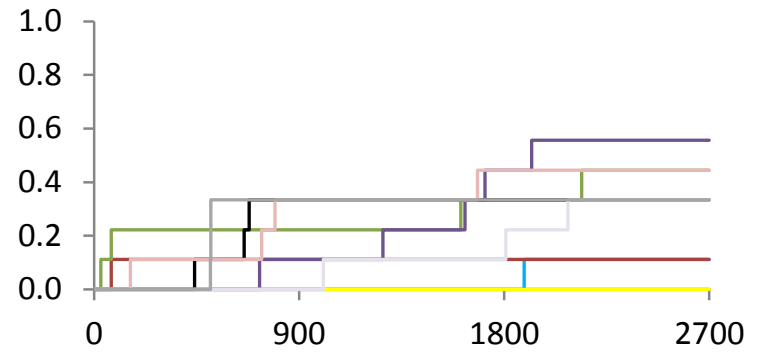

(b) 3 green light trained, 6 untrained

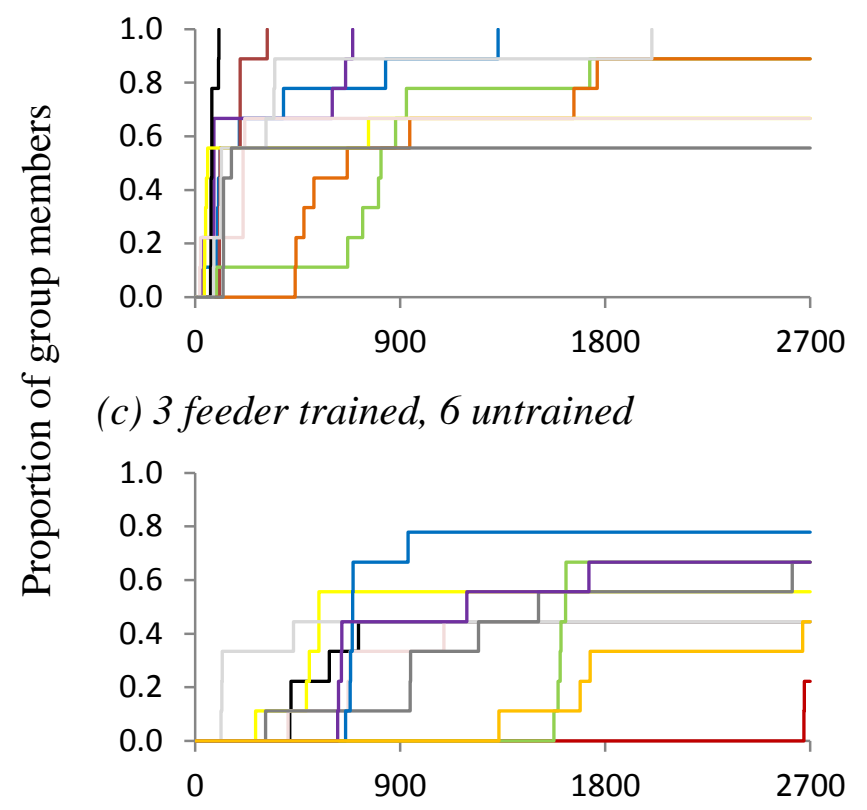

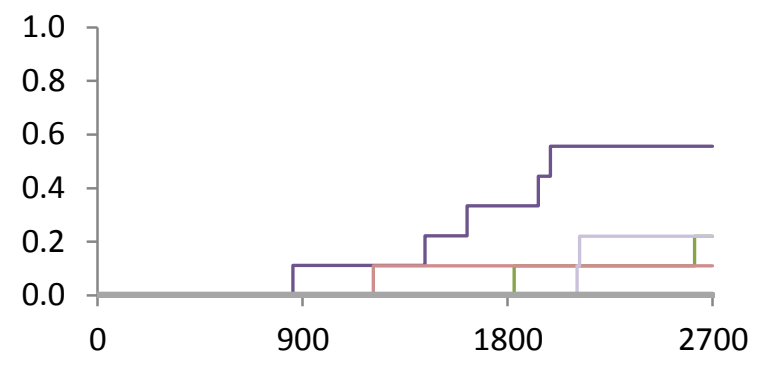
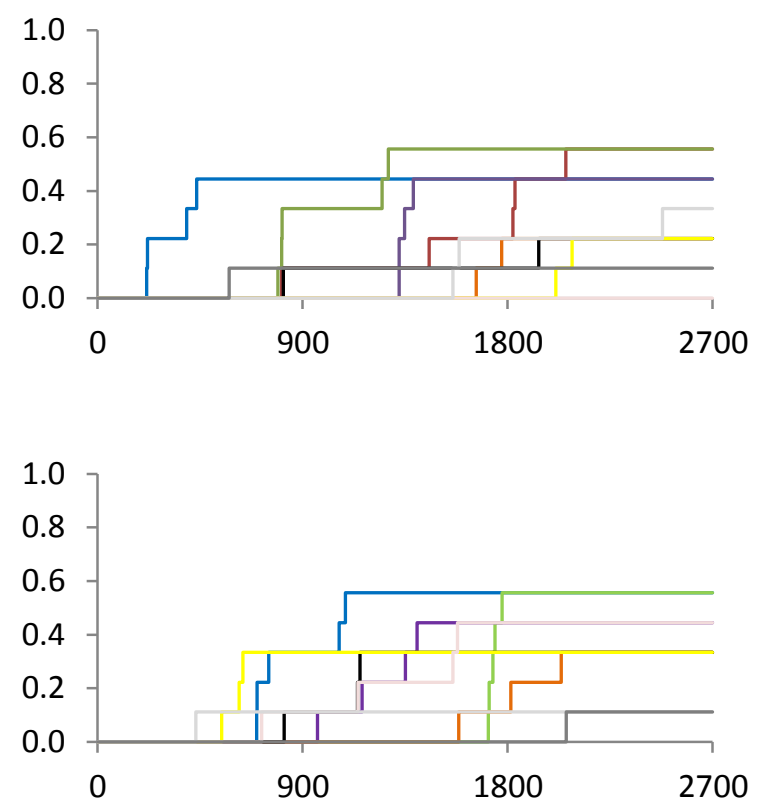

(d) 3 green light trained, 3 feeder trained, 3 untrained
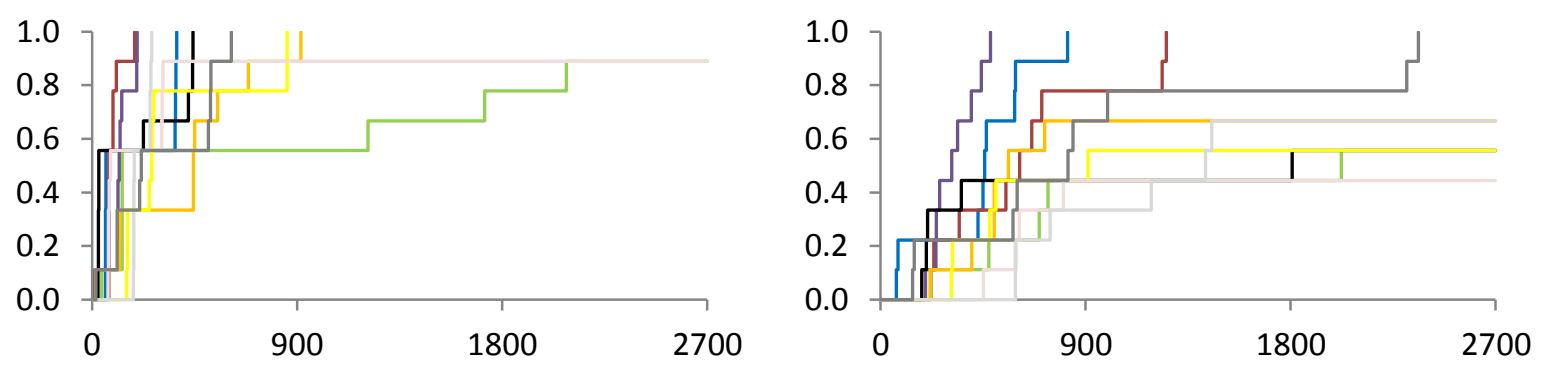

Time (s)

690 Supplementary Figure 1. Survival plots showing the time (s) that each fish first entered the green light goal zone (left panels) and the feeder (right panels). Each line represents a single 692 replicate, with the same coloured line referring to the same replicate between the left and right 693 panels. (a) - (d) present results for the four different treatments. 


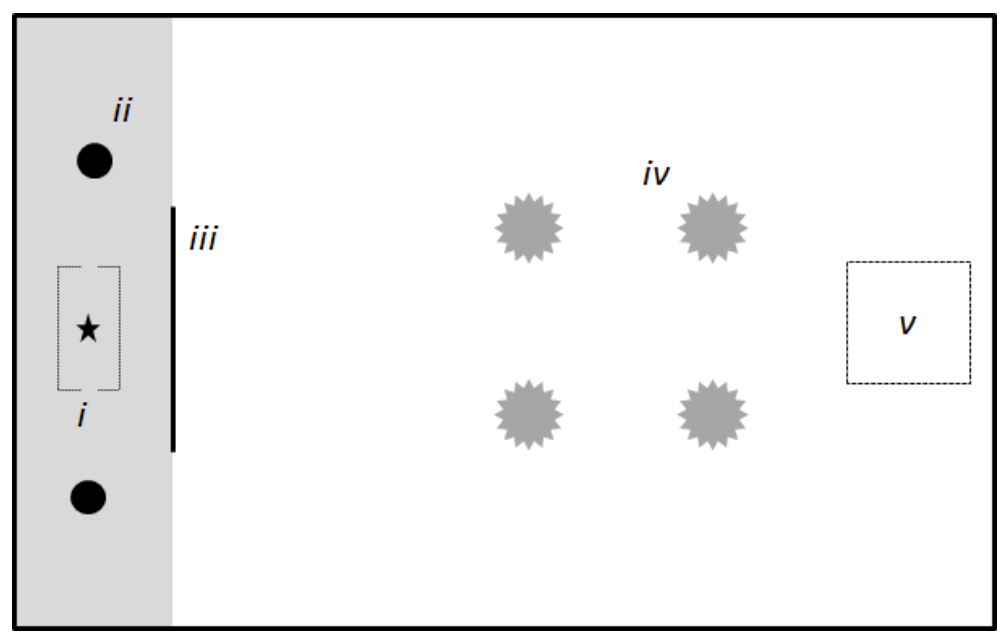

Supplementary Figure 2: The experimental arena, consisting of a feeder box $(i)$ containing a prey patch, two green lights $(i i)$, which fish in some trials had been trained to approach, an opaque screen (iii), artificial plants (iv) and a holding unit (v), within which were housed before 694 the start of the trial. See main text for further details and experimental procedure. 
(a)

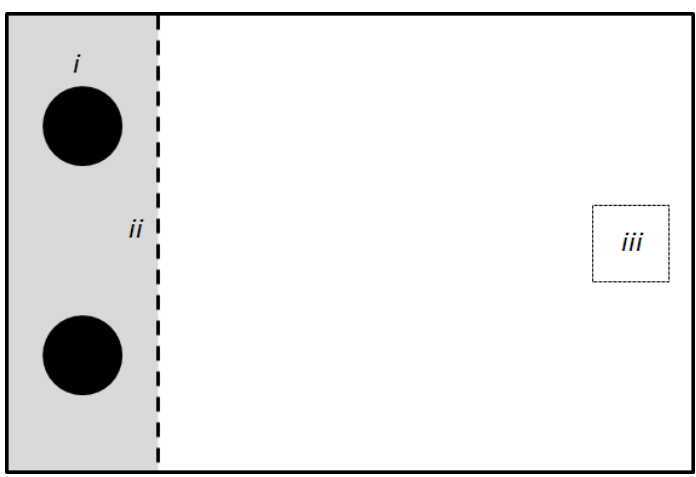

(b)

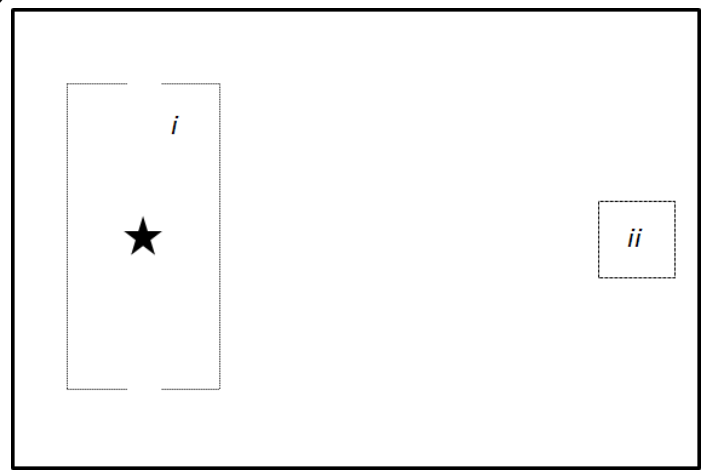

Supplementary Figure 3. The experimental areas used in the pilot experiments. (a), the light-training pilot, indicating the location of the green lights ( $i$ ), the goal zone (ii) and the holding unit (iii) used to house the fish at the start of the trial. (b), the feeder-training pilot, with the feeder unit ( $i$ ) and the holding unit (ii). See text for further details and procedure. 
(a) Green light training pilot experiment

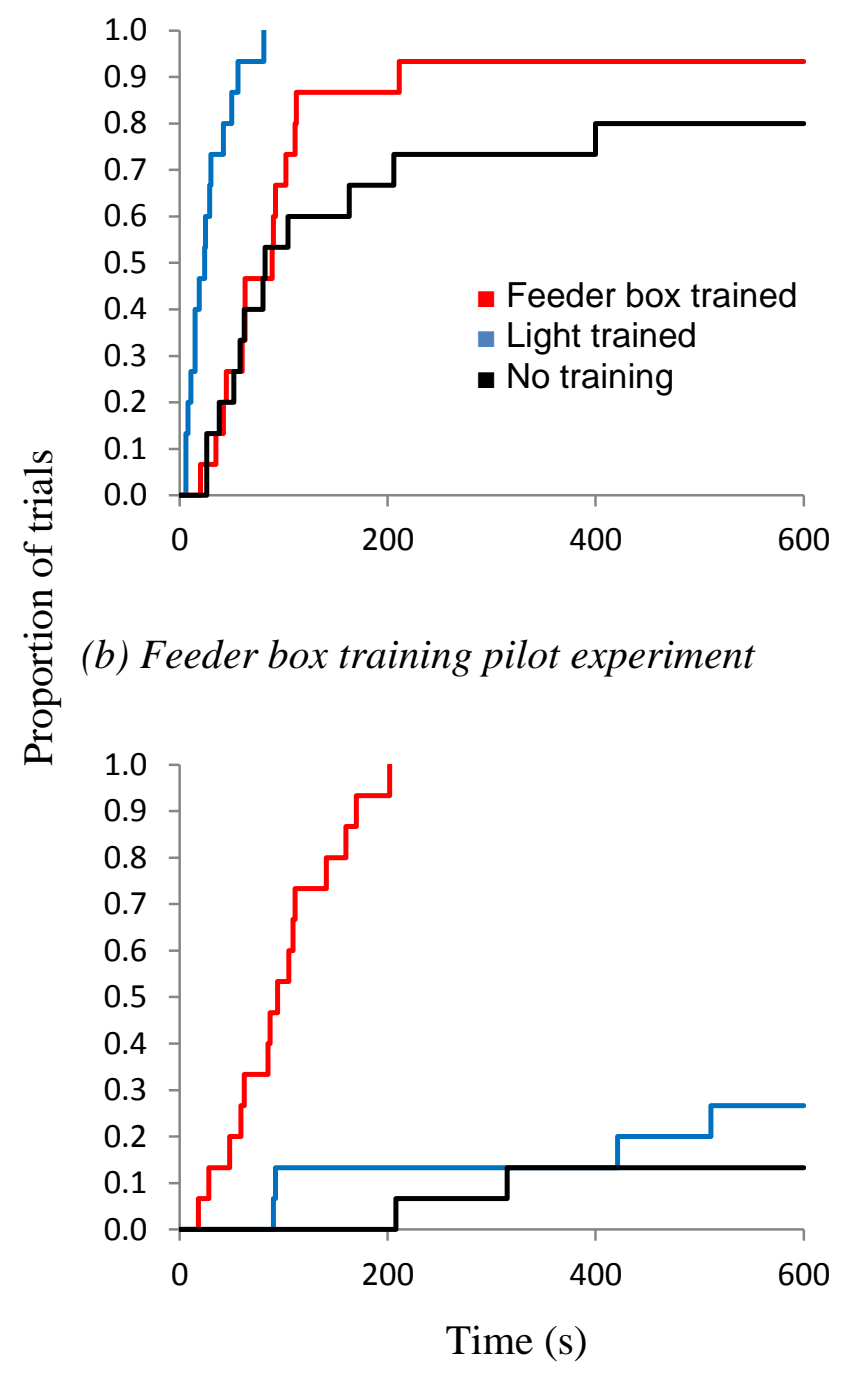

Supplementary Figure 4: The latencies of fish to enter the goal zone in the green-light training pilot (a) and the feeder box in the feeder-training pilot (b).The light- and feedertrained fish were faster in each respective experiment. 
766 Supplementary Table 1. Training batches and testing schedule. U refers to untrained fish, F to 767 feeder-trained and L to light-trained, with the number of fish in each referring to the group

768 composition of the experimental treatment. See main text for further details.

769

Batch Experiment When tested Replicates per treatment per batch

$\begin{array}{ccccccc} & & 9 \mathrm{U} & 3 \mathrm{~F}, 6 \mathrm{U} & 3 \mathrm{~L}, 6 \mathrm{U} & 3 \mathrm{~L}, 3 \mathrm{~F}, 3 \mathrm{U} \\ 2 & \text { Pilot (Light) } & \text { Sept } 2012 & - & - & - & - \\ & \text { Pilot (Feeder) } & \text { Oct } 2012 & - & - & - & -\end{array}$

Main Nov 2012

5

3b Main Nov 2012

5

Main Jan 2013

Main Jan 2013

Main

Feb 2013

Main

Feb 2013

Mar 2013

Main

Mar 2013

5

5

5

5

771

772

773

774

775

776

777

778

779

780

781

782

783

784

785

786

787

788

789

790

791

792

793

794

795

796

797

798

799 
800 Supplementary Table 2. Model coefficients for predicting the rate at which fish enter the goal 801 zone. The social cues are linear predictors based on the number of fish that fit a criteria (e.g.

802 number of fish in the goal area).

803

Variable

Experience

Social Cues, Number of...

... fish in the goal area

... entrances within $10 \mathrm{~s}$

... exists within $10 \mathrm{~s}$

... green trained light

fish

... feeder trained fish

Training

Light

Feeder

$\begin{array}{rrrr}\text { Coefficient } & \text { SE } & \boldsymbol{z} & \boldsymbol{p} \\ -0.01 & 0.01 & -1.25 & 0.21 \\ & & & \\ -0.14 & 0.05 & -2.79 & <0.01 \\ 2.13 & 0.11 & 19.25 & <0.01 \\ 0.29 & 0.60 & 0.48 & 0.63 \\ 0.43 & 0.09 & 5.05 & <0.01 \\ 0.19 & 0.06 & 3.19 & <0.01 \\ & & & \\ 1.01 & 0.18 & 5.67 & <0.01 \\ 0.31 & 0.19 & 1.64 & 0.10\end{array}$

804

805

806

807

808

809

810

811

812

813

814

815

816

817

818

819

820

821

822

823

824

825

826

827

828

829

830

831

832

833 
834 Supplementary Table 3. Model coefficients for predicting the rate at which fish enter the 835 feeder. The rates for Naïve fish are fixed to zero. The social cues are linear predictors based the 836 number of fish that fit a criteria (e.g. number of fish in the goal area).

837

\section{Variable}

Experience

Social Cues, Number of...

... fish in the goal area

... entrances within $10 \mathrm{~s}$

... exists within $10 \mathrm{~s}$

... green trained light

fish

... feeder trained fish

Training

Light

Feeder

$\begin{array}{rrrr}\text { Coefficient } & \text { SE } & \boldsymbol{z} & \boldsymbol{p} \\ -0.01 & 0.01 & -2.14 & 0.03 \\ & & & \\ -1.48 & 0.27 & -5.39 & <0.01 \\ 6.95 & 0.54 & 12.83 & <0.01 \\ 0.76 & 0.50 & 1.53 & 0.13 \\ -0.23 & 0.14 & -1.68 & 0.09 \\ 0.01 & 0.15 & 0.05 & 0.96 \\ & & & \\ 0.18 & 0.23 & 0.79 & 0.43 \\ 0.63 & 0.22 & 2.84 & <0.01\end{array}$

